\title{
Central Nervous System Angiosarcoma
}

National Cancer Institute

\section{Source}

National Cancer Institute. Central Nervous System Angiosarcoma. NCI Thesaurus. Code C5450.

A malignant vascular neoplasm arising from the brain, spinal cord or meninges. 